\title{
Aspectos reproductivos de Anodonta Luteola Lea 1858 (Unionoida: Unionidae) en Cañas, Guanacaste, Costa Rica
}

\author{
Reproductive aspects of Anodonta Luteola Lea 1858 (Unionoida: Unionidae) in \\ Cañas, Guanacaste, Costa Rica
}

\author{
Rafael Cruz-Soto \\ rcruzsoto47@gmail.com \\ Escuela de Ciencias Biológicas \\ Universidad Nacional. Puntarenas, Costa Rica \\ Rebeca Quesada-Céspedes \\ rebeca.quesada.cespedes@una.cr \\ Escuela de Ciencias Biológicas \\ Universidad Nacional. Puntarenas, Costa Rica
}

Recibido-Received: 10/dic/2015 / Corregido-Corrected: 10/jun /2016.

Aceptado-Accepted: 5/set/2016 / Publicado-Published: 31/ene /2017.

\begin{abstract}
Resumen
Se estudiaron los aspectos reproductivos de Anodonta luteola. Las muestras se obtuvieron de mayo de 2014 a abril de 2015 de un estanque de cultivo de tilapia (Oreochromis niloticus). El sexo se determinó mediante frotis de la gónada. La relación sexual se calculó dividiendo el número de hembras por el de machos y se utilizó la prueba de Chi-cuadrado para determinar sus diferencias significativas. Se determinó que $A$. luteola es dioica y la proporción sexual fue de 1.21:1 machos/hembras; sin embargo, el test Chi-cuadrado no mostró diferencias significativas de la proporción 1:1 $\left(\mathrm{X}^{2}=3.112, \mathrm{p}>0.05\right)$. Las hembras se clasificaron en no grávidas y grávidas. Se calculó el índice de condición como IC = peso fresco de la carne / (longitud x altura diámetro) x 1000. Además, se calculó el rendimiento en carne. De acuerdo con los resultados, esta especie desova durante todo el año. Los picos de desove se observaron durante los meses de junio, agosto, octubre, marzo y abril. Las temperaturas más elevadas marcaron los principales picos de desove. El índice de condición no mostró correlación con ninguno de los estados de desarrollo de las demibranquias de las hembras. El rendimiento en carne osciló entre 47\% y 51\%.
\end{abstract}

Palabras clave: Reproducción; condición; rendimiento.

\begin{abstract}
Reproductive aspects of Anodonta luteola were studied. Between May 2014 and April 2015, samples were collected from a pond culture of tilapia (Oreochromis niloticus). Sex was determined by smears of the gonad. The sex ratio was calculated by dividing the number of females by males, and Chi-square test was used to determine their significant differences. It was determined that $A$. luteola is dioecious, and sex ratio was 1.21: 1 male / female. However, the Chi-square test showed no significant difference in the proportion 1: $1\left(\mathrm{X}^{2}=3.112, \mathrm{p}>0.05\right)$. Females were classified as pregnant and non-pregnant. The condition index as $\mathrm{CI}=$ fresh meat weight / (height $\mathrm{x}$ diameter length) was calculated $\mathrm{x} 1000$. In addition, the meat yield was calculated. According to the results, this species spawns throughout the year. Spawning peaks were observed during the months of June, August, October, March and April. Higher temperatures marked the major peaks of spawning. The condition index showed no correlation with any of the development stages of demibranchs of females. The meat yield ranged between $47 \%$ and $51 \%$.
\end{abstract}

Key words: reproduction, condition, yield. 
UNICIENCIA Vol. 31, No. 1, pp. 51-57. Enero-junio, 2017.

Anodonta luteola (Lea, 1858) es un molusco bivalvo de agua dulce confundido por muchos años con Glabaris luteolus y Anodontites luteola (Arias-Andrés, 2014; Castillo-Martínez, 2000; Cruz \& Villalobos, 1984; Cruz \& Villalobos, 1985; Villalobos \& Cruz, 1984; Villalobos et al., 1984). La identificaron solo como Anomalodesmata sp. A. luteola (Ruiz, 1982; Ruiz \& Bonilla, 1982) se distribuye desde Nicaragua hasta Darién, Panamá (Von Martens 1890-1901) y está presente en ríos, embalses, estanques y de forma natural en estanques de cultivo de tilapias. Cruz \& Quesada (2016, inédito) determinaron su posición taxonómica y la estructura del gloquidio, como un miembro de la familia Unionidae, propio de la Provincia Panámica y no introducida de otras latitudes como se sospechaba (Garcés \& García, 2004). El objetivo del presente trabajo fue describir aspectos reproductivos básicos de A. luteola.

\section{Método}

Desde mayo de 2013 hasta abril de 2014 se recolectaron 500 ejemplares de A. luteola para un promedio de 45 individuos por mes. Las almejas se recolectaron a mano a $0.5 \mathrm{~m}$ de profundidad en un estanque de cultivo de tilapias de la especie Oreochromis niloticus, de la Universidad Técnica Nacional (UTN), ubicada en Cañas, Guanacaste $\left(10^{\circ} 20^{\prime} 8^{\prime}\right.$ ' N, 85 $\left.5^{\circ}{ }^{\prime} 19^{\prime \prime} \mathrm{W}\right)$. Las almejas fueron transportadas en una hielera a la Estación de Biología Marina (EBM) de la Universidad Nacional (UNA) $\left(9^{\circ} 58^{\prime} 43^{\prime \prime} \mathrm{N}, 84^{\circ} 50^{\prime} 25^{\prime \prime} \mathrm{W}\right)$.

Para determinar la relación sexual de cada ejemplar se le determinó el sexo haciendo un frotis de la gónada en los casos en los que no se encontraran gloquidios en las demibranquias. La relación sexual fue calculada dividiendo el número de hembras por el de machos y se utilizó la prueba de Chi-cuadrado para determinar diferencias estadísticamente significativas en la proporción de sexos.

Con base en Semenas \& Brugni (2002), las hembras se clasificaron en las siguientes categorías: no grávidas (grado I) y grávidas (grados II y III). Se consideraron hembras grado I a aquellas con las branquias vacías, grado II a las que presentaban desarrollo del marsupio con óvulos fecundados o con embriones sin valvas y grado III a aquellas que tenían desarrollo del marsupio con gloquidios bien desarrollados.

Durante todo el muestreo se midió la temperatura del agua en el estanque para obtener un promedio mensual. Luego a cada ejemplar se le tomaron los siguientes datos: peso total (PT) y peso fresco de la carne (PFC) a $0.01 \mathrm{~g}$; longitud total (LT) (distancia máxima del borde anterior al posterior de la valva), altura total (AT) (distancia perpendicular desde el umbo hasta el borde ventral) y diámetro o ancho (DT) (distancia máxima de la valva derecha a la izquierda). Con el fin de examinar la variación estacional en peso antes y después del desove se calculó el índice de condición utilizado por Kang et al., (2007), quienes relacionan el peso fresco de la carne con el volumen intervalvar (LT $\mathrm{x}$ AT $x$ DT), de acuerdo con la siguiente fórmula: $\mathrm{IC}=\mathrm{PFC} /\left(\mathrm{LT}^{*} \mathrm{AT}^{\star} \mathrm{DT}\right)^{\star} 1000$

Los ejemplares se agruparon en dos categorías de talla: 1(20-94mm), 2(95-168mm) para calcular el rendimiento en carne, mediante la fórmula: $\mathrm{R}=(\mathrm{PFT} / \mathrm{PT})^{\star} 100$

\section{Análisis y resultados}

Se determinó que A. luteola es dioica y que la relación sexual de machos/hembras fue de 1.21:1. El porcentaje de machos fue mayor que el de hembras durante ocho meses, excepto en los meses de octubre, enero, marzo y abril. Sin embargo, el test Chi-cuadrado no mostró diferencias estadísticamente significativas de la proporción 1:1 $\left(\mathrm{X}^{2}=3.112\right.$, p >0.05) (Cuadro1). 
ISSN Electrónico: 2215-3470

DOI: http://dx.doi.org/10.15359/ru.31-1.6
UNICIENCIA Vol. 31, No. 1, pp. 51-57. Enero-junio, 2017.

URL: www.revistas.una.ac.cr/uniciencia Email: revistauniciencia@una.cr

Tabla 1

Razón sexual (hembras/machos) de Anodonta luteola, análisis de Chi-cuadrado con el nivel de probabilidad

\begin{tabular}{|c|c|c|c|c|c|}
\hline Meses & Machos & Hembras & Razón sexual & $\mathrm{X}^{2}$ & Probabilidad \\
\hline Mayo & 22 & 14 & 1.57 & 1.778 & \\
\hline Junio & 19 & 16 & 1.18 & 0.257 & \\
\hline Julio & 27 & 3 & 2.07 & 4.900 & $\mathrm{p}<0.05\left(^{*}\right)$ \\
\hline Agosto & 25 & 18 & 1.39 & 1.140 & \\
\hline Setiembre & 27 & 19 & 1.42 & 1.391 & \\
\hline Octubre & 17 & 23 & 0.74 & 0.900 & \\
\hline Noviembre & 24 & 14 & 1.71 & 2.632 & \\
\hline Diciembre & 20 & 19 & 1.05 & 0.026 & \\
\hline Enero & 12 & 21 & 0.57 & 2.455 & \\
\hline Febrero & 26 & 14 & 1.85 & 3.600 & \\
\hline Marzo & 19 & 19 & 1.00 & 0.000 & \\
\hline Abril & 13 & 23 & 0.56 & 2.778 & \\
\hline Total & 251 & 213 & 1.21 & 3.112 & \\
\hline
\end{tabular}

Nota: El asterisco indica diferencia estadísticamente significativa. Elaboración propia de la investigación.

La talla mínima en machos y hembras con presencia de células sexuales fue de $26 \mathrm{~mm}$ y $35 \mathrm{~mm}$ respectivamente. Durante todo el periodo de estudio se encontraron hembras de $A$. luteola en los estadios de no grávidas y grávidas (Figura 1). Considerando que las hembras no grávidas han desovado, se puede notar que se manifestaron desoves todo el año excepto en el mes de julio. Los principales picos de desove ocurrieron en mayo (35.7\%), junio (37\%), agosto (38\%), octubre (50\%) marzo (47\%) y abril (70\%).

La temperatura del agua en los estanques (Figura 2) fluctuó entre $25.94^{\circ} \mathrm{C}$ y $28.75^{\circ} \mathrm{C}$, con un promedio de $27.14 \pm 0.8^{\circ} \mathrm{C}$. Los valores más altos de la temperatura resultaron en los meses de mayo, junio, agosto, octubre, marzo y abril.

El índice de condición calculado para A. luteola varió entre 0.11 y 0.15 con un promedio de $0.13 \pm 0.02$. El valor más alto resultó en el mes de mayo $(0.15)$ y el más bajo $(0.11)$ en octubre. El coeficiente de correlación calculado entre el índice de condición y los ejemplares no grávidos (grado I) resultó ser de -0.27 .

El rendimiento en carne de A. luteola resultó ser de $51 \%$ en los ejemplares de la talla 1 y de $43 \%$ para los de la talla 2 , con un promedio de $47 \%$. 
UNICIENCIA Vol. 31, No. 1, pp. 51-57. Enero-junio, 2017.

URL: www.revistas.una.ac.cr/uniciencia

Email: revistauniciencia@una.cr
ISSN Electrónico: 2215-3470

DOI: $\underline{\text { http://dx.doi.org/10.15359/ru.31-1.6 }}$

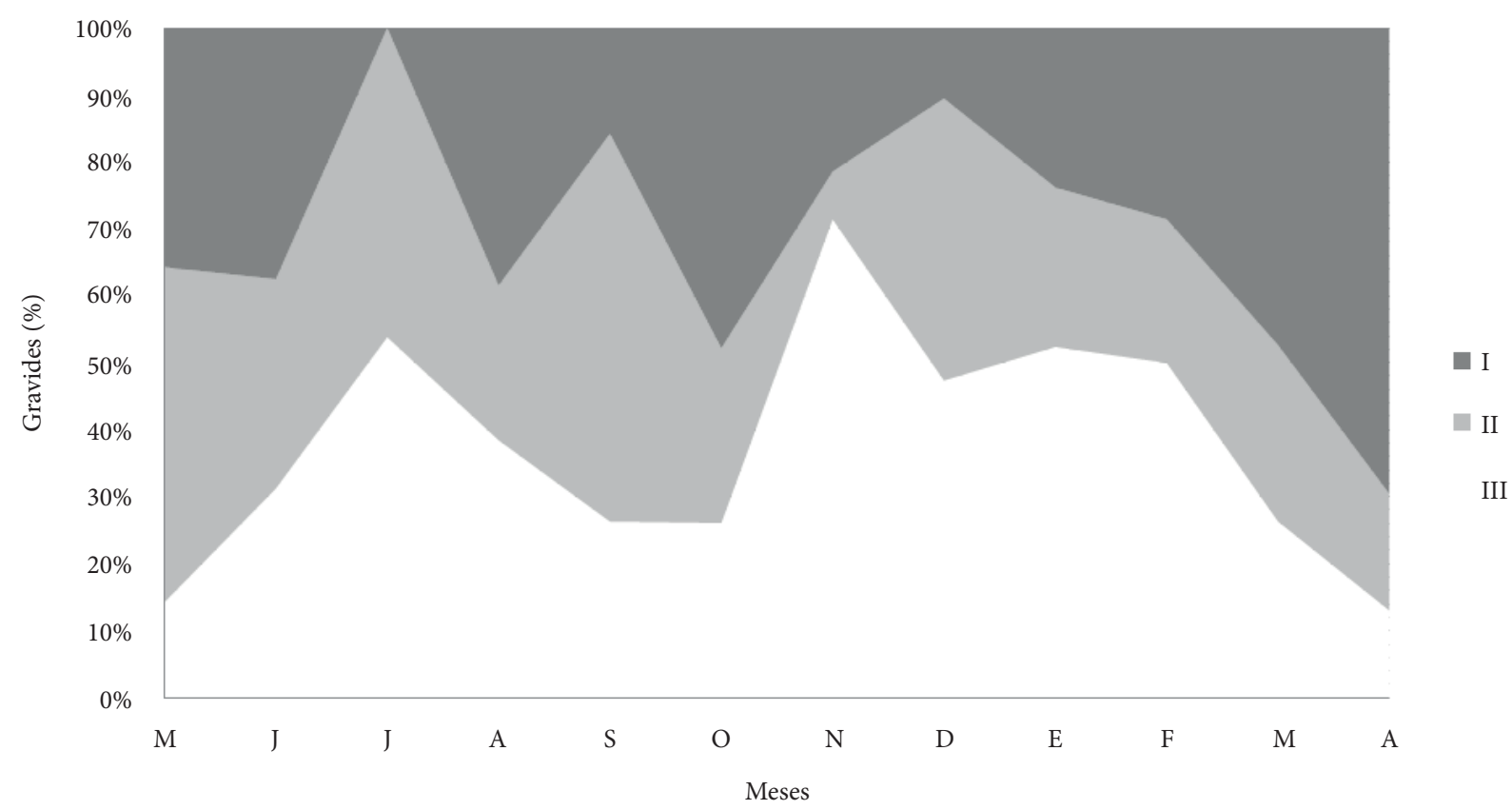

Figura 1. Variación mensual de las hembras de A. luteola no grávidas (grado I) y grávidas (grados II y III). Elaboración propia de la investigación.

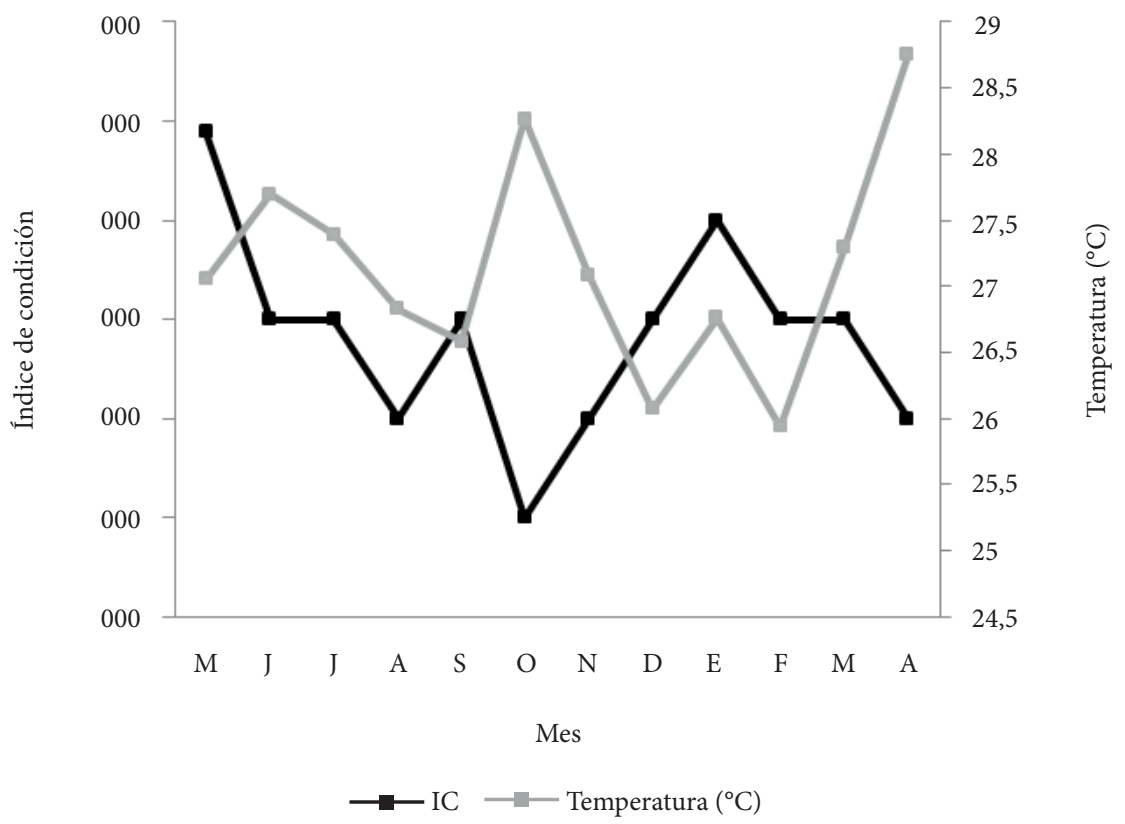

Figura 2. Variación mensual delíndice de condición medidoen A. luteolaytemperatura del agua del estanque donde crece. Elaboración propia de la investigación. 
ISSN Electrónico: 2215-3470

DOI: http://dx.doi.org/10.15359/ru.31-1.6
UNICIENCIA Vol. 31, No. 1, pp. 51-57. Enero-junio, 2017.

URL: www.revistas.una.ac.cr/uniciencia Email: revistauniciencia@una.cr

\section{Discusión}

Los machos y hembras de A. luteola mostraron equilibrio en la proporción sexual, lo que permite que cada hembra madura tenga mayor posibilidad de ser fecundada, redundando en el éxito de la reproducción. Los resultados de este estudio mostraron que las hembras de A. luteola maduran a tallas mayores que los machos, situación similar ocurre en Diplodon chilensis (Semenas \& Brugni, 2002). Según Honkoop et al. (1999), los huevos de los bivalvos contienen gran cantidad de lípidos con relativamente mayor contenido de energía que la proteína y carbohidratos, por lo tanto, de mayor costo energético. De acuerdo con Ilano et al., (2003), el uso de las reservas de la glándula digestiva para las actividades reproductivas en las hembras y no en los machos también está probablemente relacionado con la mayor inversión reproductiva en las hembras.

En A. luteola se encontró una correspondencia entre la variación de la temperatura del agua y los periodos de desove, de tal manera que las frecuencias más elevadas de organismos desovados resultaron cuando las temperaturas de los estanques fueron las más elevadas $(\mathrm{r}=0.82, \mathrm{p} \leq 0.05)$.

Los índices de condición, en la mayoría de los casos, sirven para indicar el estado de salud y bienestar y reflejan la actividad fisiológica de los moluscos bivalvos y, en muchos casos, como en Polymesoda solida (De la hoz, 2010) y Modiolus squamosus (Prieto et al., 1999), sus valores coinciden con la actividad reproductiva. En algunas especies, como en Arca zebra (Lista et al., 2008), Venerupis rhomboides (Yamusa et al., 2010) y A. luteola, en el presente trabajo, los índices utilizados no reflejaron la actividad reproductiva.

El rendimiento en carne resulta ser mayor en los bivalvos de agua dulce, debido principalmente a que las concentraciones de carbonato de calcio son mucho mayores en el mar que en los ríos o estanques de agua dulce. Por la escasa información sobre este aspecto en almejas de agua dulce, en este trabajo se hacen comparaciones con especies marinas.

En el presente trabajo se reporta un promedio de 47\% de carne total, este valor resultó ser muy superior a los reportados por Squires et al. (1978) para Anadara tuberculosa (15\%), Cabrera et al., (1995) para Modiolus capax (25.9\%), Silva \& Bonilla (2001) para Anadara similis (21.2 \%), Arriache et al. (2002) para Perna perna (29.37\%) y Acosta et al. (2011) para Perna viridis (34\%). Por tanto, se concluye que $A$. luteola resulta ser una especie apta para su cultivo, debido a su alto potencial reproductivo y su óptimo porcentaje de rendimiento en carne.

\section{Agradecimientos}

Este trabajo forma parte del proyecto código 0233-10 de la Escuela de Ciencias Biológicas, Facultad de Ciencias Exactas y Naturales, Universidad Nacional que fue posible por fondos de Ley de Pesca. Se le agradece a Cristian Fonseca Rodríguez, responsable del proyecto, por el apoyo para la toma de muestras y la realización del documento; a Jorge Boza, Rafael Araujo y Jorge Alfaro por la revisión del manuscrito; a William Vargas por haber proporcionado las almejas; al personal de la Estación experimental Jiménez Núñez, por su ayuda en la recolección; a Stephanie Rueda e Isaac Cruz, por su colaboración en el trabajo de laboratorio. 
UNICIENCIA Vol. 31, No. 1, pp. 51-57. Enero-junio, 2017.

ISSN Electrónico: 2215-3470

URL: www.revistas.una.ac.cr/uniciencia

DOI: $\underline{\text { http://dx.doi.org/10.15359/ru.31-1.6 }}$

Email: revistauniciencia@una.cr

\section{Referencias}

Acosta, R., Prieto, A., Licet, B., Longart, Y., \& Montes, M. (2011). Rendimiento, índice de condición y esfuerzo reproductivo del mejillón verde Perna viridis en cultivo de fondo en el Golfo de Cariaco, Estado Sucre, Venezuela. Zootecnia Trop., 29(4), 399-410.

Arias-Andrez, M., Mena, F., \& Pinnoch, M. (2014). Ecotoxicological evaluation of aquaculture and agriculture sediments with biochemical biomarkers and bioassays: antimicrobial potential exposure. J. Environ. Biol., 35(1),107-117.

Arriache, D., Licet, B., García, N., Lodeiros, C., \& Prieto, A. (2002). Índice de condición, gonádico y de rendimiento del mejillón marrón Perna perna (Bivalvia: Mytilidae, del Morro de Guarapo, Venezuela. INTECIENCIA, 27(11), 613-619.

Cabrera, J., Cruz, R. A., Solano, Y., \& Protti, M. (1995). Biometría de Modiolus capax (Bivalvia: Mytilidae) en Playa Ocotal, Guanacaste, Costa Rica. Rev. Biol. Trop., 45, 173-176.

Castillo-Martínez, L. E. (2000). Pesticide impact of intensive banana production on aquatic ecosystems in Costa Rica (Dissertation doctoral). Sweden: Stockholm University.

Cruz, R. A., \& Villalobos, C. R. (1984). Estudios sobre la biología de Glabaris luteolus (Mycetopodidae: Bivalvia) IV. Biometría y aspectos reproductivos en 28 millas. Limón. Costa Rica. Rev. Lat. Acuic., 21, 9-17.

Cruz, R. A., \& Villalobos, C. R. (1985). Tamaño y madurez sexual de la almeja de agua dulce Glabaris luteolus (Lea 1852) (Mycetopodidae: Bivalvia). Brenesia, 24, 371-374.

De La Hoz, M. V. (2010). Condición somática de la almeja Polymesoda solida (Veneroidea: Corbiculidae) durante el periodo lluvioso, en el Parque Natural Isla de Salamanca, Caribe Colombiano. Rev. Biol. Trop., 58 (1), 131-145

Garcés, H., \& García, J. (2004). Colecta y análisis de muestras biológicas de los Lagos Gatún y Miraflores: N. ${ }^{\circ}$ 3: Bentos. Centro de Ciencias del Mar y Limnología (CCML). Facultad de Ciencias Naturales, Exactas y Tecnología. Universidad de Panamá.

Honkoop, P., Van der Meer, J., Beukema, J. \& Kwast, D. (1999). Reproductive investment in the intertidal bivalve Macoma balthica. J. Sea Res., 41, 203-212. http://dx.doi.org/10.1016/S1385-1101(98)00053-7

Ilano, A., Fujinaga, K., \& Nakao, S. (2003). Reproductive cycle and size at sexual maturity of the commercial whelk Buccinum isaotakii in Funka Bay, Hokkaido, Japan. J. Mar. Biol. Ass. U.K., 83, 1287-1294. http://dx.doi.org/10.1017/S0025315403008683

Kang, D-H., Hyun, C-Y., Limpanont, Y. \& Choi, K-S. (2007). Annual gametogenesis of the Chinese anapela clam Coecella chinensis (Deshayes 1855) at an upper intertidal sandy beach on the east coast of Jeju, Korea. J. Shellfish Res, 26(2), 433-441. http://dx.doi.org/10.2983/0730-8000(2007)26[433:AG OTCA]2.0.CO;2

Lista, M., Prieto, A., Velásquez, C., Lodeiros, C. \& Hernández, G. (2008). Variación mensual del índice de condición y madurez sexual en la pepitona Arca zebra, del banco de Chacopata, Península de Araya, Estado de Sucre, Venezuela. Saber. 20(1), 29-38.

Prieto, A. S., Flores, M. \& Lodeiros, C. (1999). Madurez sexual e índice de condición en una población del mejillón de fondo Modiolus squamosus (Mollusca, Bivalvia) en Tocuchare, Golfo de Cariaco, Venezuela. ECOTROPICOS, 12(2), 83-90.

Ruiz, R. \& Bonilla, R. (1982). Intentan su reproducción en policultivo con peces. ASBANA, 6(16), 20

Ruiz, R. (1982). Ensayo de crecimiento en policultivo. ASBANA, 6(17), 6-7.

Semenas, L., \& Brugni, N. (2002). Características poblacionales y ciclo de vida de Diplodon chilensis (d.Orbigny, 1835) (Hyriidae, Bivalvia) en el lago Gutiérrez (Patagonia, Argentina). Ecología Austral, $12,29-40$. 
ISSN Electrónico: 2215-3470

DOI: http://dx.doi.org/10.15359/ru.31-1.6
UNICIENCIA Vol. 31, No. 1, pp. 51-57. Enero-junio, 2017. URL: www.revistas.una.ac.cr/uniciencia Email: revistauniciencia@una.cr

Silva-Benavides, A.-M., \&. Bonilla-Carrion, R. (2001). Abundancia y morfometría de Anadara tuberculosa y A. similis (Mollusca: Bivalvia) en el Manglar de Purruja, Golfo Dulce, Costa Rica. Rev. Biol. Trop., $49,315-320$.

Squires, H. J., Esteves, M., Barona, O. \& Mora, O. (1975). Mangrove Cockles, Anadara spp. of the Pacific Coast of Colombia. The Veliger, 18, 57-68.

Villalobos, C. R., \& Cruz, R. A. (1984). Biología de Glabaris luteolus (Mycetopodidae: Bivalvia). I. Distribución de tamaño, crecimiento y mortalidad en Cañas, Guanacaste, Costa Rica. Rev. Biol. Trop., 32, 29-34.

Villalobos, C. R., Cruz, R. A., y Báez, A. L. (1984). Biología de Glabaris luteolus (Mycetopodidae: Bivalvia) III. Distribución de tamaño, crecimiento y mortalidad en 28 Millas, Limón, Costa Rica. Rev. Biol. Trop., 32, 57-60.

Von Martens, E. (1890-1901). Biologia Centrali- Americana. Land and freshwater Mollusca. London: Ed. R.H. Porter and Dulai.

Yamuza-Clavijo, M. P., Rodríguez-Rúa, A. \&. Bruzón-Gallego, M. A. (2010). Ciclo gametogénico de Venerupis rhomboides Pennant, 1777 en el litoral Andaluz (sur España) Hidrobiológica, 20(3), 195-202.

\section{@() $\Theta \Theta \Theta$}

Aspectos reproductivos de Anodonta Luteola Lea 1858 (Unionoida: Unionidae) en Cañas, Guanacaste, Costa Rica (Rafael Cruz-Soto y otros) por Revista Uniciencia se encuentra bajo una Licencia CreativeCommons Atribución-NoComercialSinDerivadas 3.0 Unported. 